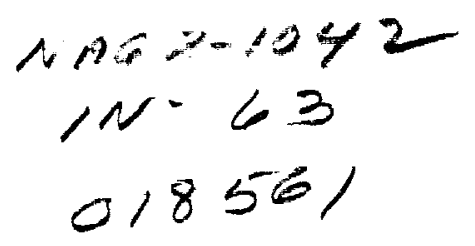

\title{
Preview-Based Stable-Inversion for Output Tracking
}

\author{
Qingze Zou * Santosh Devasia ${ }^{\dagger}$
}

\begin{abstract}
Stable Inversion techniques can be used to achieve high-accuracy output tracking. However, for nonminimum phase systems, the inverse is noncausal - hence the inverse has to be precomputed using a pre-specified desired-output trajectory. This requirement for pre-specification of the desired output restricts the use of inversion-based approaches to trajectory planning problems (for nonminimum phase systems). In the present article, it is shown that preview information of the desired output can be used to achieve online inversion-based output tracking of linear systems. The amount of preview-time needed is quantified in terms of the tracking error and the internal dynamics of the system (zeros of the system). The methodology is applied to the online output tracking of a flexible structure and experimental results are presented.
\end{abstract}

*Graduate Student, Mechanical Engineering Department, University of Utah, SLC, UT, 84112. Email: qingze@eng.utah.edu

${ }^{\dagger}$ Assistant Professor, Mechanical Engineering Department, University of Utah, SLC, UT, 84112. Email: santosh@eng.utah.edu 


\section{Introduction}

Inversion of system dynamics can be used to find inputs which achieve exact output-tracking $[10,22]$. For systems with nonminimum phase dynamics, inputs found through standard inversion techniques tend to be unbounded and therefore, cannot to be used for practical outputtracking. Recently, stable inversion approaches $[5,11,1]$ have been developed for nonminimum phase systems, which yield bounded inputs for exact output-tracking. The application of such inversion-based output tracking has been studied for several nonminimum systems, like flexible manipulators $[20,16]$, aircraft systems $[18,24,4]$, and high precision positioning of piezo-probes for nano-technology [2]. A critical difficulty with these inversion-based approaches is that the inverse is noncausal (for non-minimum phase systems) and therefore the desired output trajectory must be pre-specified. This requirement for pre-specification of the output-trajectory can be a substantial limitation on the use of the non-causal inversion-based approach, and it limits the inversion-based approach to trajectory planning applications. The main contribution of this paper is to show that, for linear systems, the noncausal inverse can be computed using a preview-based approach, which allows the inversion process to be applied online. This article also quantifies the amount of preview-time needed in terms of the desired tracking accuracy and the location of the zeros of the system. Implementation issues are discussed and the approach is experimentally verified by applying it to the output tracking of a flexible structure with nonminimum phase dynamics.

A major result in output tracking is the solution of the output regulation problem for linear systems by Francis [9]. These results were generalized for the nonlinear case by Isidori and Byrnes [13]. The desired outputs are assumed to be generated by an exosystem and the linear regulator is easily designed by solving a manageable set of linear equations. A problem, however, with the regulator approach is that the exosystem states are often switched to describe 
the desired output; this leads to transient tracking errors after the switching instants. Such switching-caused transient errors can be avoided by using inversion-based approaches to output tracking $[5,7]$. Thus, it is advantageous to use inversion-based output tracking when exacttracking of a particular output trajectory is required. In the inversion based approach, the system dynamics $[10,22]$ is inverted to find the input that exactly tracks a single specified output trajectory (rather than track a class of outputs as in the case of the output-regulator). Inversion for nonminimum phase systems is challenging since the standard approaches lead to unbounded inputs [10]. Stable inversion techniques resolve this problem of unbounded inverse-inputs by finding bounded (but possibly noncausal) exact-tracking input-state trajectories $[5,11]$. The noncausality of the inverse implies that the entire output trajectory needs to be known ahead of time which restricts the use of inversion-based approaches (for nonminimum phase systems). This motivates the current work, which shows that the noncausal inverse can be found by using preview information and thereby enables the online implementation of the inversionbased output tracking technique for nonminimum phase systems.

Other approaches have also used preview information of the desired output trajectory for output tracking, for example, to alleviate the problems due to nonminimum phase dynamics by exploiting actuator redundancy [25]. Another use of previewed information of the output is in linear quadratic-based (lq-based) optimal output tracking controllers (see [17], Chapter 4). Tomizuka and coworkers (e.g., [23]) have shown that the performance of finite-time-preview controllers approach the performance of the infinite-preview controller as the amount of preview time increases. In these works, the goal is to trade-off the tracking requirement to reduce the magnitude of the inputs. In contrast, inversion based approaches aim to achieve high accuracy control of the desired output trajectory. Trading off the accuracy output-tracking requirement to achieve other goals like vibration minimization is also possible within the inversion-based framework (see [8]). The resulting inverse controller is noncausal, which can also be imple- 
mented using the preview-based controller discussed in the current article.

The paper is organized in the following format. The inversion-based output tracking scheme and its dependence on the solution of the system's internal dynamics is presented in Section 2. The inversion problem is then solved using preview information of the output, and implementation issues are studied in Section 3. In section 4, the preview-based inversion approach is applied to the output tracking control of a flexible structure and experimental results are presented. Discussions are in section 5 and our conclusions are in Section 6.

\section{Stable Inversion for Nonminimum Phase Systems}

In this Section, the inversion-based output tracking approach is presented. It is shown that solving the inversion problem is equivalent to finding bounded solutions to the systems's internal dynamics.

\subsection{Output tracking using inversion of system dynamics}

Consider a linear system described by

$$
\begin{aligned}
\dot{x}(t) & =A x(t)+B u(t) \\
y(t) & =C x(t)
\end{aligned}
$$

which has the same number of inputs as outputs, $u(t), y(t) \in \Re^{p}$, and $x(t) \in \Re^{n}$. We assume that the system is stabilizable. Let $y_{d}(\cdot)$ be the desired output trajectory to be tracked. Then in the inversion-based approach we, first, find a nominal input-state trajectory, $\left[u_{f f}(\cdot), x_{r e f}(\cdot)\right]$ that satisfies the system equations (1) and yields the desired output exactly, i.e.,

$$
\left.\begin{array}{rl}
\dot{x}_{r e f}(t) & =A x_{r e f}(t)+B u_{f f}(t) \\
y_{d}(t) & =C x_{r e f}(t)
\end{array}\right\} \quad \forall t \in(-\infty, \infty)
$$


and, second, we stabilize the exact-output yielding state trajectory, $x_{\text {ref }}(\cdot)$, by using state feedback (see Figure 1). Thus $x(t) \rightarrow x_{r e f}(t)$ and $y(t) \rightarrow y_{d}(t)$ as $t \rightarrow \infty$ and output tracking is achieved. While stabilization of the reference state trajectory can be easily achieved through standard techniques [14] like state feedback of the form $K\left[x(t)-x_{\text {ref }}(t)\right]$, the main challenge is to find the inverse input-state trajectory $\left[u_{f f}(\cdot), x_{r e f}(\cdot)\right]$ - especially for systems with nonminimum phase dynamics. This paper addresses the on-line computation of the inverse input-state trajectory using preview information of the desired trajectory, $y_{d}$.

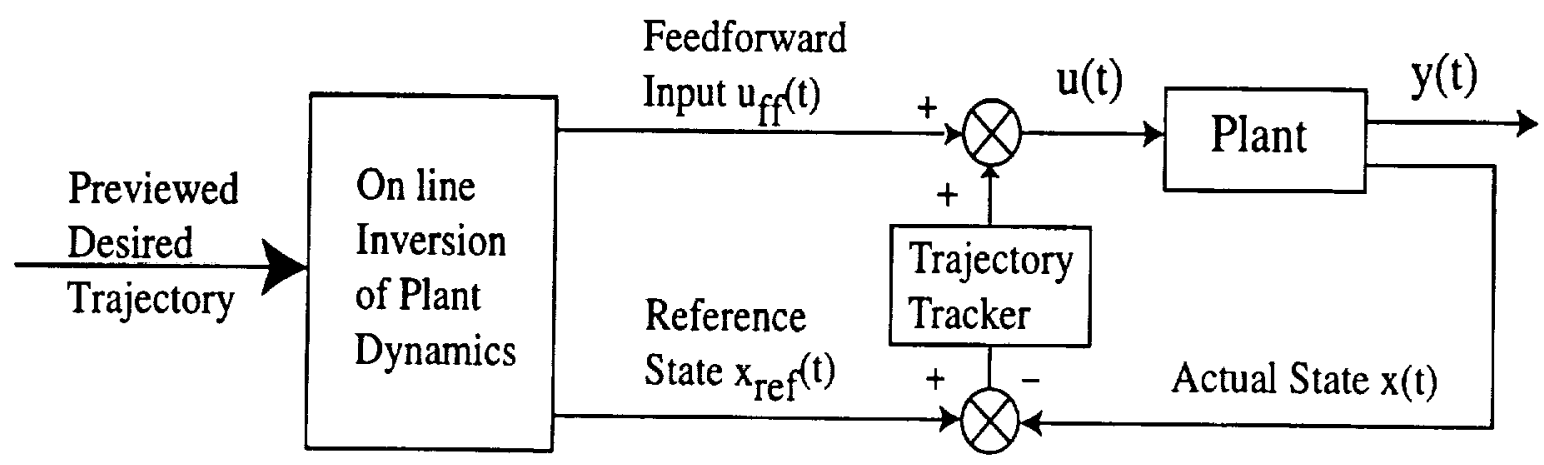

Figure 1. The output tracking control scheme.

\subsection{Stable inversion scheme}

In this subsection, it is shown that finding the inverse input-state trajectory is equivalent to finding bounded solutions to the system's internal dynamics. Let the linear system (1) have a well defined vector relative degree, $r:=\left[r_{1}, r_{2}, \ldots, r_{p}\right]$. Then the output's derivatives are given as:

$$
\frac{d^{r_{k}} y_{k}}{d t^{r_{k}}}=C_{k} A^{r_{k}} x+C_{k} A^{r_{k}-1} B u
$$


where $C_{k}$ is the $k^{\text {th }}$ row of $C$, and $1 \leq k \leq p$. In vector notation let equation (3) be rewritten as

$$
y^{(r)}(t)=A_{x} x(t)+B_{y} u(t)
$$

where

$$
\begin{aligned}
& y^{(r)}:=\left[\begin{array}{llll}
\frac{d^{r_{1}} y_{1}}{d t^{r_{1}}} & \frac{d^{\tau_{2}} y_{2}}{d t^{r_{2}}} & \ldots & \frac{d^{r_{p}} y_{p}}{d t^{r_{p}}}
\end{array}\right]^{T} \\
& A_{x}:=\left[\begin{array}{c}
C_{1} A^{r_{1}} \\
C_{2} A^{r_{2}} \\
\vdots \\
C_{p} A^{r_{p}}
\end{array}\right] ; \quad B_{y}:=\left[\begin{array}{c}
C_{1} A^{r_{1}-1} B \\
C_{2} A^{r_{2}-1} B \\
\vdots \\
C_{p} A^{r_{p}-1} B
\end{array}\right],
\end{aligned}
$$

and $B_{y}$ is invertible because of the well-defined relative degree assumption. Equation (4) motivates the choice of the control law of the form

$$
u_{f f}(t)=B_{y}^{-1}\left[y_{d}^{(r)}(t)-A_{x} x(t)\right]
$$

for all $t \in(-\infty, \infty)$. Substituting this control law in equation (4), it is seen that exact tracking is maintained, i.e.,

$$
y^{(r)}(t)=y_{d}^{(r)}(t)
$$

To study the effect of this control law consider a change of coordinates $T$ such that

$$
\left[\begin{array}{l}
\xi(t) \\
\eta(t)
\end{array}\right]=T x(t)
$$

where $\xi(t)$ consists of the output and its time-derivatives

$$
\xi(t):=\left[y_{1}, \dot{y}_{1}, \ldots, \frac{d^{r_{1}-1} y_{1}}{d t^{r_{1}-1}}, y_{2}, \dot{y}_{2}, \ldots, \frac{d^{r_{2}-1} y_{2}}{d t^{r_{2}-1}}, \ldots, y_{p}, \dot{y}_{p}, \ldots, \frac{d^{r_{p}-1} y_{p}}{d t^{\tau_{p}-1}}\right]^{\prime} .
$$


The system equation (1) can then be re-written in the new-coordinates as

$$
\begin{aligned}
\dot{\xi}(t) & =\hat{A}_{1} \xi+\hat{A}_{2} \eta+\hat{B}_{1} u \\
\dot{\eta}(t) & =\hat{A}_{3} \xi+\hat{A}_{4} \eta+\hat{B}_{2} u
\end{aligned}
$$

where

$$
\hat{A}:=T A T^{-1}:=\left[\begin{array}{cc}
\hat{A}_{1} & \hat{A}_{2} \\
\hat{A}_{3} & \hat{A}_{4}
\end{array}\right] ; \text { and } \hat{B}:=\left[\begin{array}{c}
\hat{B}_{1} \\
\hat{B}_{2}
\end{array}\right]=T B
$$

In the new coordinates, the control law for maintaining exact tracking (Equation (5)) can be written as

$$
u_{f f}(t)=B_{y}^{-1}\left[y_{d}^{(r)}(t)-A_{\xi} \xi_{d}(t)-A_{\eta} \eta(t)\right]
$$

where

$$
\left[\begin{array}{c}
A_{\xi} \\
A_{\eta}
\end{array}\right]:=A_{x} T^{-1} \text {. }
$$

Note that the desired $\xi(\cdot)$ is known when the desired output trajectory $y_{d}(\cdot)$ and the output's time derivatives are specified. This desired $\xi(\cdot)$ is defined as $\xi_{d}(\cdot)$. Since the control law was chosen such that exact tracking is maintained, $y^{(r)}(t)=y_{d}^{(r)}(t)$ we also have $\dot{\xi}(t)=\dot{\xi}_{d}(t)$, and Equations (7) and (8) become

$$
\begin{aligned}
\dot{\xi}(t) & =\dot{\xi}_{d}(t) \\
\dot{\eta}(t) & =\hat{A}_{3} \xi_{d}(t)+\hat{A}_{4} \eta(t)+\hat{B}_{2} B_{y}^{-1}\left[y_{d}^{(r)}(t)-A_{\xi} \xi_{d}(t)-A_{\eta} \eta(t)\right] \\
& :=\hat{A}_{\eta} \eta(t)+\hat{B}_{\eta} \mathbb{Y}_{d}(t)
\end{aligned}
$$

where

$$
\hat{A}_{\eta}:=\hat{A}_{4}-\hat{B}_{2} B_{y}^{-1} A_{\eta} ; \quad \hat{B}_{\eta}:=\left[\begin{array}{ll}
\hat{B}_{2} B_{y}^{-1} & \hat{A}_{3}-\hat{B}_{2} B_{y}^{-1} A_{\xi}
\end{array}\right] ; \quad \text { and } \mathbb{Y}_{d}:=\left[\begin{array}{c}
y_{d}^{\tau}(t) \\
\xi_{d}(t)
\end{array}\right]
$$


This is the inverse system, and in particular, Equation (11) represents the internal dynamics. If a bounded solution, $\eta_{d}(\cdot)$, to the internal dynamics (11) can be found then the exact-tracking feedforward input can be found through equation (9) as

$$
u_{f f}(t)=B_{y}^{-1}\left[y_{d}^{(r)}(t)-A_{\xi} \xi_{d}(t)-A_{\eta} \eta_{d}(t)\right]
$$

and the reference state trajectory can be found as

$$
x_{r e f}(t)=T^{-1}\left[\begin{array}{c}
\xi_{d}(t) \\
\eta_{d}(t)
\end{array}\right] .
$$

Thus a bounded solution to the internal dynamics (11) is required to find the inverse for applying the output tracking scheme shown in Figure 1.

\subsection{Bounded solutions to the internal dynamics}

We restrict the following discussion to systems with hyperbolic internal dynamics, i.e., none of the zeros of the system (Equation 1) lies on the imaginary axis of the complex plane (a technique to address the nonhyperbolic case can be found in [4]). If the internal dynamics is hyperbolic, there exists a transformation $U$ such that the internal dynamics (11) can be decoupled into a stable subsystem $\left(\sigma_{s}\right)$ and an unstable subsystem $\left(\sigma_{u}\right)$ :

$$
\begin{aligned}
& \dot{\sigma}_{s}(t)=\tilde{A}_{s} \sigma_{s}(t)+\tilde{B}_{s} \mathbb{Y}_{d}(t) \\
& \dot{\sigma}_{u}(t)=\tilde{A}_{u} \sigma_{u}(t)+\tilde{B}_{u} \mathbb{Y}_{d}(t)
\end{aligned}
$$

where

$$
\sigma(t):=\left[\begin{array}{c}
\sigma_{s}(t) \\
\sigma_{u}(t)
\end{array}\right]=U \eta(t)
$$

Bounded solution to the internal dynamics in the transformed coordinates can then be found as 


$$
\begin{gathered}
\sigma_{s}(t)=\int_{-\infty}^{t} e^{\bar{A}_{s}(t-\tau)} \tilde{B}_{s} \mathbb{Y}_{d}(\tau) d \tau \\
\sigma_{u}(t)=-\int_{t}^{\infty} e^{-\tilde{A}_{u}(\tau-t)} \tilde{B}_{u} \mathbb{Y}_{d}(\tau) d \tau
\end{gathered}
$$

In the new coordinates, the feedforward control law in Equation (13) can be written as:

$$
u_{f f}(t)=B_{y}^{-1}\left[y_{d}^{r}(t)-A_{\xi} \xi_{d}(t)-A_{\eta} \hat{U}_{s} \sigma_{s}(t)-A_{\eta} \hat{U}_{u} \sigma_{u}(t)\right]
$$

where $U^{-1}:=\left[\hat{U}_{s} \hat{U}_{u}\right]$ is partitioned according to the partition of $\sigma$ in Equation (17).

This completes the inversion technique. To summerize: the bounded solutions found through Equations (18) and (19) are used to find a bounded solution to the internal dynamics, $\eta_{d}$, by using Equation (17). The inversion is then completed by finding the reference state and input trajectories by using Equations (14) and (20), which are then used in the control scheme shown in Fig. 1 to obtain output tracking. Note that only the past information is needed to compute the solution to the stable subsystem of the internal dynamics by using Equation (18). However, to find a bounded solution to the unstable subsystem $\left(\sigma_{u}\right)$ by using Equation (19), the desired output must be completely specified (including future information). This is the main problem, which restricts the use of inversion to trajectory planning (where it may be acceptable to solve Equation (19) off-line).

\section{Preview Based Inversion}

In this section, we discuss the online computation and implementation of the inverse using preview-information of the desired output trajectory, which enables the tracking of output trajectories that are specified on-line. We begin by quantifying the relationship between the preview-time and tracking error. 
Let the desired output, $y_{d}$ (and its time-derivatives) be given for a preview-time of $T_{p}$ seconds, i.e., at time $t, \mathbb{Y}_{d}(\tau)$ (defined in Equation (12)) is known for all $t \leq \tau \leq t+T_{p}$. This preview information is used to approximate the solution to the internal dynamics (in particular, to approximate the bounded solution to the unstable subsystem, $\sigma_{u}$, given by Equation 19). The approximated solution, $\tilde{\sigma}_{u}$ is found as

$$
\tilde{\sigma}_{u}(t)=-\int_{t}^{t+T_{p}} e^{-\tilde{A}_{u}(\tau-t)} \tilde{B}_{u} \mathbb{Y}_{d}(\tau) d \tau
$$

Let the error between the exact-solution to the unstable subsystem found through Equation (19) and the approximated-solution found through Equation (21) be defined as:

$$
e_{\sigma_{u}}=\sigma_{u}(t)-\tilde{\sigma}_{u}(t)
$$

We show in the following Lemma that $e_{\sigma_{u}}(t)$ can be made arbitrarily small by having a large enough preview time. Furthermore, we show that the error in computation of the inverse input by using the finite preview also converges to zero as the preview time, $T_{p}$, increases. The error in computing the inverse-input, $e_{u}(t)$, is defined as

$$
e_{u}(t)=\tilde{u}_{f f}(t)-u_{f f}(t)
$$

where $u_{f f}(t)$ denotes the input obtained with infinite preview using Equation (20), and $\tilde{u}_{f f}(t)$ denotes the preview input obtained with finite preview when $\sigma_{u}$ is replaced by the approximate solution $\tilde{\sigma}_{u}$ in Equation (20)

$$
\tilde{u}_{f f}(t):=B_{y}^{-1}\left[y_{d}^{r}(t)-A_{\xi} \xi_{d}(t)-A_{\eta} \hat{U}_{s} \sigma_{s}(t)-A_{\eta} \hat{U}_{u} \tilde{\sigma}_{u}(t)\right] .
$$

This implies that, as the preview time increases, the approximated input trajectory approaches (arbitrarily closely) the exact output-tracking inverse trajectory.

Lemma 1 Let the desired trajectory and its derivatives be bounded, i.e. there exists a positive scalar $M \in \mathbb{R}$, such that $\left\|\mathbb{Y}_{d}(t)\right\|_{2} \leq M$ for all time $t$. Then for any scalar $\varepsilon>0$, there 
exists a finite time $T_{p}^{*}$ such that the error (in computing the inverse input by using the preview information of the output)can be made smaller than $\varepsilon$ if the preview time is larger than $T_{p}^{*}$, i.e., $\left\|e_{u}(t)\right\|_{2} \leq \varepsilon$. In the above notation, $\|z\|_{2}$ is defined as the standard Euclidean norm for any vector $z$.

Proof: Since the subsystem (16) is unstable, $-\tilde{A}_{u}$ is Hurwitz, and therefore positive scalars $\alpha$ and $\kappa$ can be chosen such that [3]

$$
\left\|e^{-\tilde{A}_{u} t}\right\|_{2} \leq \kappa e^{-\alpha t} \quad \forall t>0
$$

where, given a matrix $F \in \mathbb{R}^{n \times n},\|F\|_{2}$ denotes the induced matrix norm, defined as $\|F\|_{2}:=\sup _{\|x\|_{2}=1}\|F x\|_{2}$. From the definition of $e_{\sigma_{u}}$ in Equation (22)

$$
\begin{aligned}
\left\|e_{\sigma_{u}}(t)\right\|_{2} & =\left\|\sigma_{u}(t)-\tilde{\sigma}_{u}(t)\right\|_{2} \\
& =\left\|\int_{t+T_{p}}^{\infty} e^{-\bar{A}_{u}(\tau-t)} \tilde{B}_{u} \mathbb{Y}_{d}(\tau) d \tau\right\|_{2} \quad \text { (using Equations (19) and (21)) } \\
& \leq \int_{t+T_{p}}^{\infty}\left\|e^{-\bar{A}_{u}(\tau-t)}\right\|_{2}\left\|\bar{B}_{u} Y_{d}(\tau)\right\|_{2} d \tau \\
& \leq K_{1} \int_{t+T_{p}}^{\infty}\left\|e^{-\bar{A}(\tau-t)}\right\|_{2} d \tau \quad \text { (with } K_{1}:=M\left\|\tilde{B}_{u}\right\|_{2} \text { ) } \\
& \leq \frac{K_{1} \kappa}{\alpha} e^{-\alpha T_{p}} \quad \text { (using Equation (25)) }
\end{aligned}
$$

From the definition of the computational-error in finding the inverse-input (Equation (23)) and from Equations (20), (22) and (24), we have:

$$
\begin{aligned}
\sup _{t}\left\|e_{u}(t)\right\|_{2} & \leq \sup _{t}\left\|B_{y}^{-1}\right\|_{2}\left\|A_{\eta}\right\|_{2}\left\|\hat{U}_{u}\right\|_{2}\left\|e_{\sigma_{u}}(t)\right\|_{2} \\
& :=\sup _{t} K_{2}\left\|e_{\sigma_{u}}(t)\right\|_{2} \quad\left(K_{2}:=\left\|B_{y}^{-1}\right\|_{2}\left\|A_{\eta}\right\|_{2}\left\|\hat{U}_{u}\right\|_{2}\right) \\
& \left.\leq \frac{\mathcal{K}}{\alpha} e^{-\alpha T_{p}}, \quad \text { (using inequality (26) and } \mathcal{K}:=K_{1} K_{2} \kappa\right)
\end{aligned}
$$


For any given $\varepsilon>0$, choosing

$$
T_{p}^{*} \geq \frac{\ln \frac{\mathcal{K}}{\varepsilon \alpha}}{\alpha} .
$$

and substituting any $T_{p}>T_{p}^{*}$ in Equation (27) completes the proof.

It is noted that for proving this lemma, it is sufficient for $\left\|\tilde{B}_{u} \mathbb{Y}_{d}(\cdot)\right\|_{2}$ to be bounded, which can be less restrictive than requiring $\left\|\mathbb{Y}_{d}(\cdot)\right\|_{2}$ to be bounded. Using similar arguments, it is also possible to show that the error in computing the inverse reference-state-trajectory, $x_{r e f}$, can be made arbitrarily small by having sufficiently large preview time.

The next lemma shows that the error in output-tracking, due to errors in computing the inverse, can also be made small by choosing a sufficiently large preview time (for a similar argument, see [6])

Lemma 2 Let the original system (1) be stable (or stabilized with feedback before the inversion is applied). Then the tracking error, on applying the finite-preview-based input ( $\left.\tilde{u}_{f f}\right)$ as a feedforward input, can be made arbitrarily small by choosing a large enough preview time.

Proof: From Eq. (1), the dynamics of the state-error $e_{x}(t):=x(t)-x_{r e f}(t)$, where $x(t)$ is the system-state when the finite preview input $\tilde{u}_{f f}(t)$ is applied to system (1), can be described by

$$
\dot{e}_{x}(t)=A e_{x}(t)+B e_{u}(t)
$$

The error in output-tracking $e_{y}(t):=y(t)-y_{d}(t)=C e_{x}(t)$ can then be bounded as

$$
\begin{aligned}
& \left\|e_{y}(t)\right\|_{2}=\left\|C e_{x}(t)\right\|_{2} \leq\|C\|_{2}\left\|e_{x}(t)\right\|_{2} \\
& \leq\|C\|_{2} \int_{-\infty}^{t}\left\|e^{A(t-\tau)}\right\|_{2}\|B\|_{2}\left\|e_{u}(\tau)\right\|_{2} d \tau \text { (using solution of Equation (29)) } \\
& \leq\|C\|_{2}\|B\|_{2}\left(\sup _{\tau}\left\|e_{u}(\tau)\right\|_{2}\right) \int_{-\infty}^{\ell}\left\|e^{A(t-\tau)}\right\|_{2} d \tau
\end{aligned}
$$

If the original system(Equation(1) is stable (or if the inversion is carried out after stabilizing the system), then there exit real positive numbers $M_{1}$ and $\beta$ such that $\left\|e^{A(t-\tau)}\right\|_{2} \leq M_{1} e^{-\beta(t-\tau)}$. 
Substituting this in Equation (30) yields

$$
\begin{aligned}
& \left\|e_{y}(t)\right\|_{2} \leq\|C\|_{2}\|B\|_{2}\left(\sup _{\tau}\left\|e_{u}(\tau)\right\|_{2}\right) \int_{-\infty}^{t} M_{1} e^{-\beta(t-\tau)} d \tau \\
& =M_{2}\left(\frac{M_{1}}{\beta}\right)\left(\sup _{\tau}\left\|e_{u}(\tau)\right\|_{2}\right) \quad \text { (with } M_{2}:=\|C\|_{2}\|B\|_{2} \text { ) } \\
& \leq \frac{M_{1} M_{2} \mathcal{K}}{\alpha \beta} e^{-\alpha T_{p}} \quad \text { (using inequality (27)) }
\end{aligned}
$$

The tracking error, $\left\|e_{y}(t)\right\|_{2}$, can be kept smaller than $e_{\max }$ for all time, $t$, by choosing the preview time, $T_{p}$, as

$$
T_{p} \geq T_{p}^{*} \geq \frac{\ln \frac{M_{1} M_{2} \mathcal{K}}{\alpha \beta e_{\max }}}{\alpha}
$$

which completes the proof

Remark: The preview time $T_{p}$ needed to achieve a desired accuracy in output tracking depends on the distance of the right half plane zeros of the system (Equation (1)) from the imaginary axis of the complex plane. As this distance, increases, $\alpha$ in Equation (32) can be chosen larger (defined in Equation (25)) and therefore, a smaller preview-time can be chosen to achieve the desired accuracy in output-tracking.

\section{Example: Flexible Structure Control}

The experimental flexible structure considered here consists of two discs which are connected by a thin freely rotating shaft as shown in Figure (2). The system input, $U(t)$, is the voltage (Volts) applied to a DC motor, and the output, $\theta_{2}$, is the angular rotation (in degrees) of the disc which is farther from the motor. The system equations (obtained experimentally with a HP3562A Dynamic Signal Analyzer) can be written in the following state-space form where the state vector, $x$ is chosen as

$$
x:=\left[\begin{array}{llll}
\theta_{1} & \dot{\theta}_{1} & \theta_{2} & \dot{\theta}_{2}
\end{array}\right]^{T}
$$




$$
\begin{aligned}
& \dot{\mathbf{x}}=\left[\begin{array}{cccc}
0 & 1 & 0 & 0 \\
-3.656 & -0.436 & 3.573 & -0.091 \\
0 & 0 & 0 & 1 \\
3.245 & -0.126 & -3.259 & -0.076
\end{array}\right] x+\left[\begin{array}{c}
0 \\
21.9027 \\
0 \\
3.588
\end{array}\right] u \\
& :=A x+B u \\
& y:=\theta_{2}=\left[\begin{array}{llll}
0 & 0 & 1 & 0
\end{array}\right] x
\end{aligned}
$$

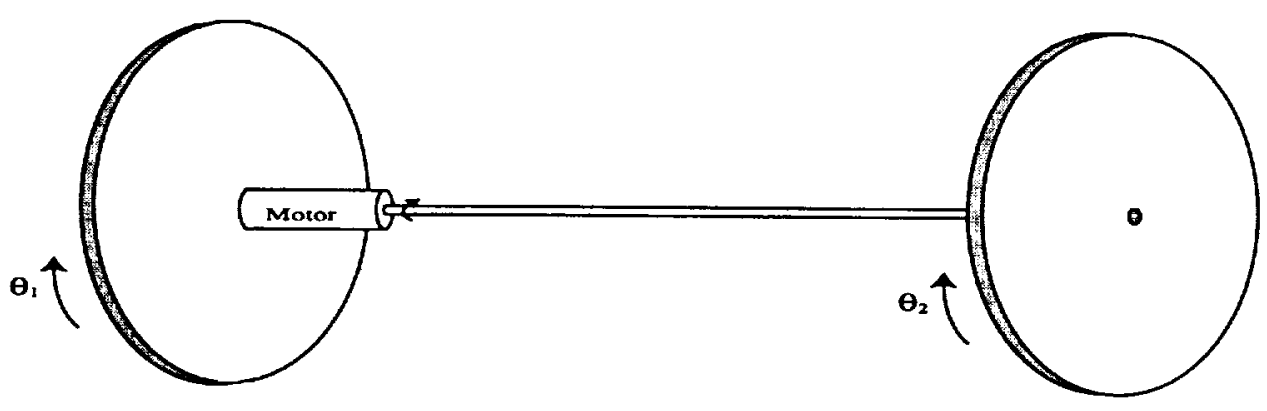

Figure 2. The experimental flexible structure.

\subsection{System inverse}

The relative degree of the above system is two and hence the output has to be differentiated twice to relate the input and the output (as described in Section (2.2) with $\xi:=[y, \dot{y}]^{T}=\left[\theta_{2}, \dot{\theta}_{2}\right]^{T}$ ). Note that $\xi$ is known when preview information of the desired output and its derivatives are defined. The internal dynamics of the system are described by $\eta:=\left[\theta_{1}, \dot{\theta}_{1}\right]^{T}$. The inverse input $u_{f f}$ can be written as (using Equation (13) and the last row of Equation (33))

$$
u_{f f}(t)=\frac{1}{3.588}\left\{\ddot{y}_{d}(t)-[3.245-0.126] \eta(t)-[-3.259-0.076] \xi_{d}(t)\right\}
$$

and the internal dynamics is given by substituting this control law into the first two rows of Equation (33) 


$$
\begin{aligned}
\dot{\eta} & =\left[\begin{array}{cc}
0 & 1 \\
-23.4658 & 0.3306
\end{array}\right] \eta+\left[\begin{array}{ccc}
0 & 0 & 0 \\
23.4658 & 0.3753 & 6.1041
\end{array}\right] \mathbb{Y}_{d} \\
& :=A_{\eta} \eta+B_{\eta} \mathbb{Y}_{d}
\end{aligned}
$$

where, $\mathbb{Y}_{d}=\left[\begin{array}{lll}y_{d} & \dot{y}_{d} & \ddot{y}_{d}\end{array}\right]^{T}$ and the entire internal dynamics is unstable, i.e., $\sigma_{u}=\eta$.

\section{2 $\quad \mathbb{Y}_{d}$ generation}

There are several methods available to generate the desired trajectory $y_{d}$ and its time derivatives. One method is to predict the future $y_{d}$ by using polynomial extrapolations of the past desired trajectory signals [19] and then differentiating these polynomials. Another approach is to use a command generator [23] and then switching its states to generate online changes of the desired trajectory. For our experimental system, we assumed that the future desired trajectory $y_{d}$ could be obtained for the preview time $T_{p}$ (a potentiometer was adjusted to define the desired output). Note that to compute the inversion-based control, the output's time derivatives must also be specified. Although these derivatives, $\dot{y}_{d}, \ddot{y}_{d}$, could be found by direct differentiation, we avoided noise problems associated with direct differentiation by using a biquad filter [12] shown in Figure 3. This filter was chosen with a cut-off frequency of $1 \mathrm{~Hz}$ to match the modelled bandwidth of the flexible structure - only the first two modes of the flexible structure were modeled and the higher-frequency dynamics are not represented by Equation (33) - see [8] for details of the flexible structure modeling. 


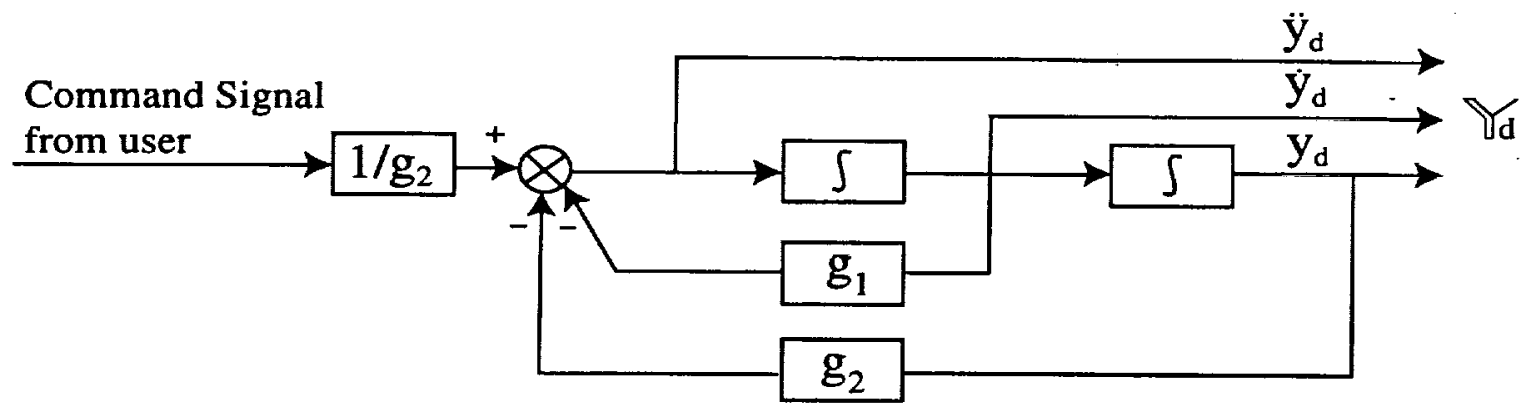

Figure 3. On-line generation of the desired trajectory and its time derivatives [12].

\subsection{Online implementation of preview-Based inversion}

The preview-based solution to the unstable subsystem of the internal dynamics, i.e., the integral Equation (21), was computed online by discretization by using the fourth-order Simpson formula [15]

$$
\begin{aligned}
\tilde{\sigma}_{u}(t) & =-\int_{t}^{t+T_{p}} e^{-\tilde{A}_{u}(\tau-t)} \tilde{B}_{u} \mathbb{Y}_{d}(\tau) d \tau \\
& \approx \frac{h}{3} \sum_{i=0}^{N} \mathcal{M}(i) \mathbb{Y}_{d}(t, i)
\end{aligned}
$$

where the sampling time is $T_{s}$ such that $N:=T_{p} / T_{s}$ is an integer, $\mathbb{Y}_{d}(t, i)=\mathbb{Y}_{d}\left(t+i * T_{s}\right)$ and:

$$
\mathcal{M}(i)= \begin{cases}-e^{-\bar{A}_{u}} \tilde{B}_{u} & \text { if } i=0 \\ -2 * e^{-\bar{A}_{u}\left(i * T_{s}\right)} \tilde{B}_{u} & \text { if } i>0 \text { and } i \text { is even } \\ -4 * e^{-\tilde{A}_{u}\left(i * T_{s}\right)} \tilde{B}_{u} & \text { if } i \text { is odd }\end{cases}
$$


Note that the matrices $\mathcal{M}(i)$ can be precomputed and stored to reduce the online computations. This computation-scheme is shown in Figure 4. The computation-error, due to timediscretization, can be reduced by choosing a small enough sampling time $\left(T_{s}\right)$.

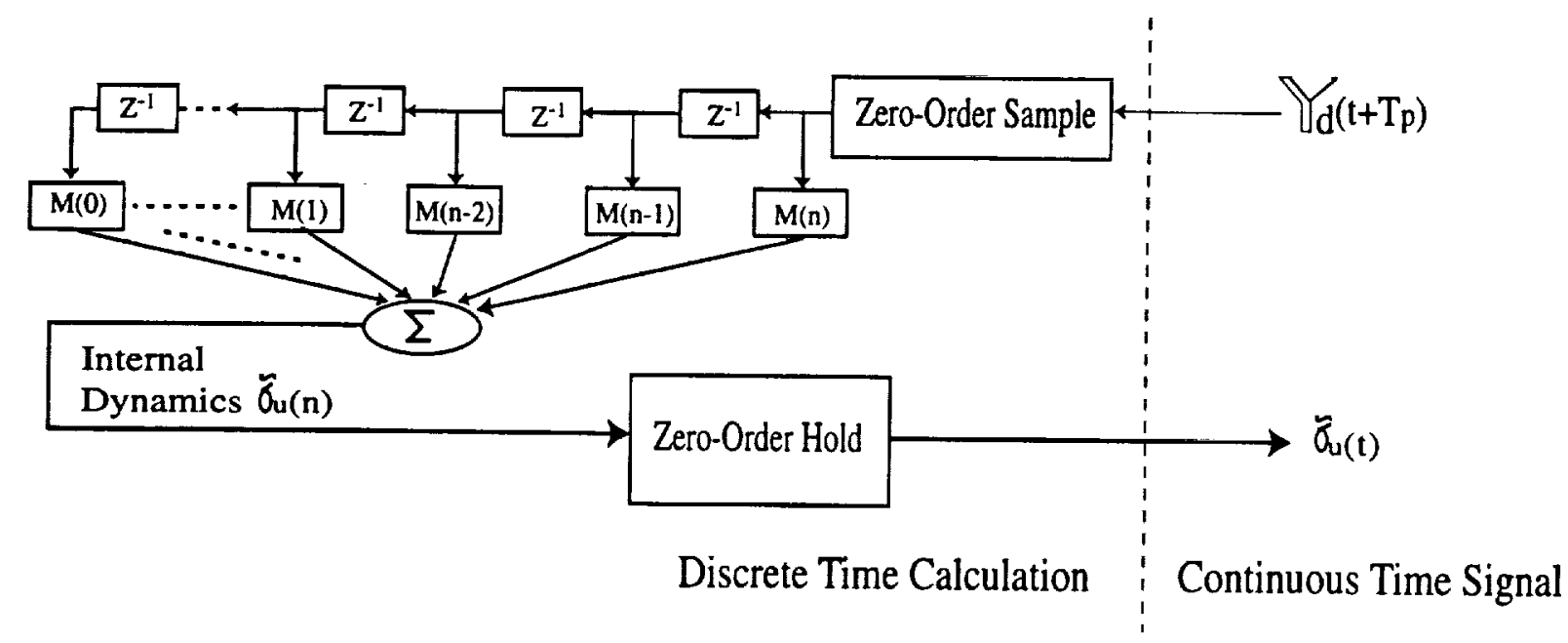

Figure 4. The schematic of on-line calculation of the unstable part of internal dynamics.

\subsection{Experimental Results}

Experimental results for output-tracking with two-different preview-time, $T_{p}=20$ seconds and $T_{p}=50$ seconds, are shown in Figure 5, which illustrates the improvement in output-tracking as preview-time increases. The experiments include feedback-stabilization, which was added to account for unmodeled dynamics in the system (like friction in the experimental system and static imbalance of the discs, which created a tendency in the discs to settle in a specific orientation). It is noted that trajectories for the two cases are different in Figure 5 because these desired output trajectories were generated on-line for the two different preview-time cases. As shown in Figure 5, preview-based inversion improves tracking performance with increasing preview time, and online specification of the output trajectory is possible. 

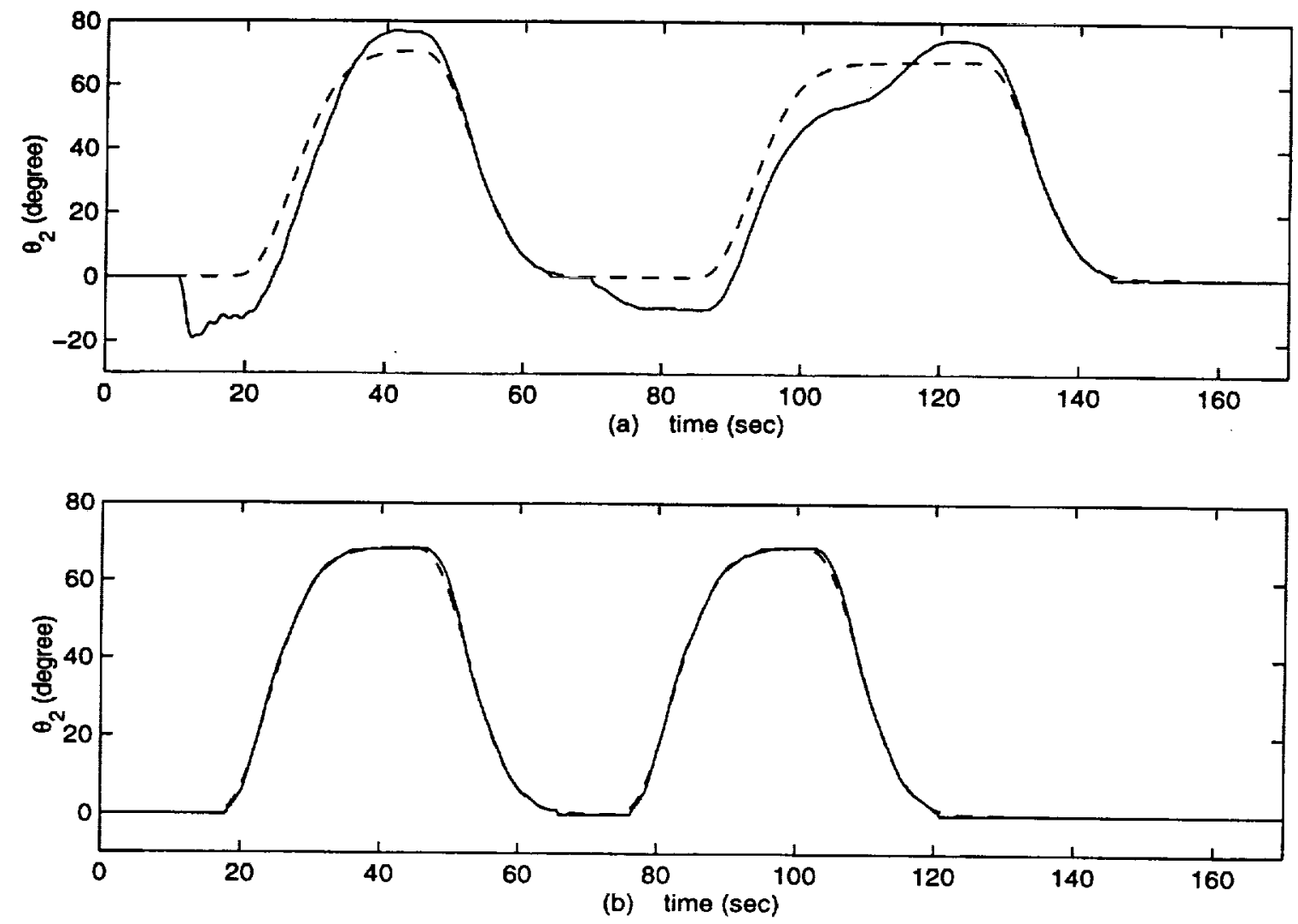

Figure 5. Experimental results. The solid line is the desired trajectory, the dashed line represents the output trajectory. Plot (a) is for 20 seconds preview time, and plot (b) is for 50 seconds preview time.

\section{Discussion}

For nonminimum phase systems, recent stable inversion-based approaches can be used to achieve high-accuracy output tracking. However, the entire desired output-trajectory has to be prescribed for the off-line computation of the inverse - this is a significant limitation since the 
desired trajectory cannot be changed online. This limitation has been alleviated by the current preview-based approach which allows the online implementation of the inversion-based outputtracking controllers. The methodology allows the application of the inversion-based approach to systems like flexible manipulators and servo-positioning systems where it is necessary to change the desired trajectory. Such online-changes are allowed by the current technique if a preview of the desired trajectory is possible. This requirement for preview information is not a drawback of the current controller. Rather, this is necessary for high-accuracy output tracking because there are performance limitations on output-tracking for nonminimum phase systems if output-preview is not available [21]. An important result of the preview-based inversion approach is that the output-tracking error can be made arbitrarily small with sufficiently large preview time. The quantification of the amount of preview time in terms of the location of system-zeros in the imaginary plane(see section 3) can aid in the initial design of a system. In particular, the preview time needed can be made smaller by choosing design-parameters such that right-half plane zeros (if any) are far away from the imaginary axis of the complex plane. In summary, the preview-based approach will help in the implementation of inversion-based control laws for high-accuracy, on-line output-tracking of nonminimum phase systems.

\section{Conclusion}

We have developed and implemented preview-based output tracking using on-line inversion for non-minimum phase systems. The preview time needed was quantified in terms of the required accuracy in output tracking, and related to the system-zeros. Implementation issues were discussed and the technique was verified by applying it to the output tracking of an experimental flexible structure. 


\section{Acknowledgment}

Financial support from NASA Ames Research Center Grant NAG 2-1042 and NSF Grant CMS 9813080 are gratefully acknowledged.

\section{References}

[1] E. Bayo. A finite-element approach to control the end-point motion of a single-link flexible robot. Journal of Robotic Systems, 4(1):63-75, 1987.

[2] D. Croft, S. Stilson, and S. Devasia. Optimal tracking of piezo-based nano-positioners. Nanotechnology, 1998. Accepted for Publication.

[3] C.A. Desoer and M. Vidyasagar. Feedback Systems: Input-Output Properties. Academic Press, 111 Fifth Avenue,N.Y. 10003, 1975. (Chapter 2).

[4] S. Devasia. Output tracking with nonhyperbolic and near nonhyperbolic internal dynamics: Helicopter hover control. Journal of Guidance, Control, and Dynamics, 20(3):573-580, 1997.

[5] S. Devasia, D. Chen, and B. Paden. Nonlinear inversion-based output tracking. IEEE Transactions on Automatic Control, 41(7):930-943, 1992.

[6] S. Devasia and B. Paden. Stable inversion for nonlinear nonminimum-phase time-varying systems. IEEE Transactions on Automatic Control, 43(2):283-288, Feb. 1999.

[7] S. Devasia, B. Paden, and C. Rossi. Exact-output tracking theory for systems with parameter jumps. International Journal of Control, 67(1):117-131, 1997. 
[8] J.S. Dewey, K. Leang, and S. Devasia. Experimental and theoretical results in outputtrajectory redesign for flexible structures. ASME Journal of Dynamic Systems, Measurement, and Control, 120:456-461, Dec. 98.

[9] B. A. Francis. The linear multi-variable regulator problem. Journal of Control Optimization, 15:486-505, 1977.

[10] R. M. Hirschorn. Invertibility of multivariable nonlinear control system. IEEE Transactions on Automatic Control, 24(6):855-865, 1979.

[11] L. R. Hunt, G. Meyer, and R. Su. Noncausal inverses for linear systems. IEEE Transactions on Automatic Control, 41(4):608-611, 1996.

[12] G. Robert Irvine. Operational Amplifier Characteristics and Applications. Prentice-Hall., Englewood Cliffs, NJ 07632, New York, 1981. (Chapter 9).

[13] A. Isidori and C. I. Byrnes. Output regulation of nonlinear systems. IEEE Transactions on Automatic Control, 35(2):131-140, 1990.

[14] H. K. Khalil. Nonlinear Systems. Prentice Hall, Upper Saddle River, NJ 07458, second edition, 1996.

[15] D. Kincaid and W. Cheney. Numerical Analysis. Brooks/Cole, Pacific Grove, CA, 93950, 1991. (Chapter 7).

[16] D. Kwon and W. J. Book. An inverse dynamic method yielding flexible manipulator state trajectories. In Proceedings of the American Control Conference, 1990.

[17] F. L. Lewis and V. L. Syrmos. Optimal Control. John Wiley and Sons, New York, second edition, 1995. 
[18] G. Meyer, L. R. Hunt, and R. Su. Nonlinear system guidance in the presense of transmission zero dynamics. NASA Technical Memorandun, (4661), Jan. 1995.

[19] R. B. Miller and M. Pachter. Maneuvering flight control with actuator constraints. Journal of Guidance, Control, and Dynamics, 20(4):729-734, 1997.

[20] B. Paden, D. Chen, R. Ledesma, and E. Bayo. Exponentially stable tracking control for multi-joint flexible manipulators. ASME Journal of Dynamic Systems, Measurement, and Control, 115(1):53-59, 1993.

[21] L. Qiu and E. J. Davison. Performance limitations of non-minimum phase systems in the servomechanism problem. Automatica, 29:337-349, March 1993.

[22] M. Silverman, L. Inversion of multivariable linear systems. IEEE Transactions on Automatic Control, 14(3):370-376, 1969.

[23] M. Tomizuka. On the design of digital tracking controllers. ASME Journal of Dynamic Systems, Measurement, and Control, 115:412-418, June 1993.

[24] C. Tomlin, J. Lygeros, and S. Sastry. Output tracking for a nonminimum phase dynamic ctol aircraft model. Controls and Decision Conference, pages 1867-1872, 1995.

[25] W. Yin and S. N. Singh. Nonlinear inverse and predictive end point trajectory control of flexible macro-micro manipulators. ASME Journal of Dynamic Systems, Measurement, and Control, 119(3):412-420, Sept. 1997. 